\title{
Source UVX par génération d'harmoniques d'ordre élevé : applications potentielles à la physique des surfaces
}

\author{
D. Riedel, J.L. Hernandez-Pozos, K.W. Kolasinski', S. Baggott ${ }^{1}$ et R.E. Palmer \\ Nanoscale Physics Research Laboratory, School of Physics and Astronomy, The University \\ of Birmingham, Edgbaston, Birmingham B15 2TT, U.K. \\ ${ }^{1}$ School of Chemistry, The University of Birmingham, Edgbaston, Birmingham B15 2TT, U.K.
}

\begin{abstract}
Résumé: Depuis la première observation de rayonnement UVX par création d'harmoniques d'ordre èlevé (HHG) dans un jet de gaz rare, la création d'impulsions laser ultra brèves dans l'UV lointain et le VUV est un domaine d'étude en perpétuel développement. La croissante facilité d'accès à des sources laser femtoseconde et à leurs amplificateurs (CPA) commerciaux a permis de nombreux progrès dans la compréhension des phénomènes fondamentaux, dans sa réalisation et mise au point, ainsi que lors de premières tentatives d'utilisation de ce type de source en spectroscopic. Le projet Birmingham Bright Light Source (BBLS) est basé sur cette idée et ouvre de nouvelles potentialités afin de faciliter l'accès à une source de type 'synchrotron'. Divers systèmes sont prêts à être étudiés, dans une seconde chambre ultravide, tels que $\mathrm{O}_{2} /$ graphite (HOPG) ainsi que $\mathrm{SF}_{6}$ ou $\mathrm{CCl}_{4} /$ silicium (100). La région d'énergie créée convient parfaitement à l'étude de petites molécules de ce type où différents processus de photo désorption pourront être observés.
\end{abstract}

\section{INTRODUCTION}

L'étude de la photo désorption de molécules physisorbées ou chimisorbées dans le domaine de la physique de la surface est en constante demande d'outils compétitifs de diagnostiques et aide à leur développement. Pour de tels systèmes, les quelques études ayant été menées sur synchrotron ont souvent négligé d'explorer le domaine d'énergie $10 \mathrm{eV}-60 \mathrm{eV}$ où il existe plusieurs molécules simples, absorbant ce type de radiation et particulièrement bien caractérisées thermodynamiquement lorsqu'elles sont adsorbées à la surface de certains matériaux. De plus, l'énergie des photons utilisés, dépasse la fonction de travail du substrat où les photoélectrons crées peuvent conduire à des processus dynamiques avec le film moléculaire adsorbé. L'idée originale d'utiliser une source cohérente UVX impulsionelle accordable basée sur la création d'harmoniques d'ordre élevé $[1,2]$ dans ce domaine de recherche est basée sur le projet Birmingham Bright Light Source. Il allie l'utilisation d'un outil puissant délivrant des trains d'impulsions ultra courtes UVX à une chambre ultra vide équipée d'outils de diagnostiques adaptés à la physique de la surface. Nous avons utilisé deux gaz rares (xénon, argon) pour caractériser la source UVX où nous avons comparé les spectres expérimentaux obtenus aux descriptions théoriques [3, 4]. Nous décrivons ensuite la méthode de mesure du flux absolu de photons obtenu après monochromateur et les mesures réalisées sous synchrotron pour $\mathrm{O}_{2} / \mathrm{HOPG}$ et $\mathrm{CO} / \mathrm{HOPG}$ que nous désirerons comparer aux résultats obtenus lors de l'utilisation de cette source.

\section{Expérience}

La figure 1 décrit la partie expérimentale de la source UVX (i) connectée à la chambre ultra vide (ii) via un monochromateur.

(i) Les lasers commerciaux utilisés (Spectra Physics) se composent d'un oscillateur Ti:Saphire (600 $\mathrm{mW}$ à $800 \mathrm{~nm}$ ) pompé par un laser à argon ionisé. Cet oscillateur est injecté dans l'étendeur temporel de faisceau de l'amplificateur régénérateur suivit de deux étages amplificateurs. Les cristaux de chaque étage sont pompés par, respectivement $10 \mathrm{~mJ}$ et $13 \mathrm{~mJ}$ à $527 \mathrm{~nm}$, rayonnement délivré par un laser YLF doublé (Spectra 


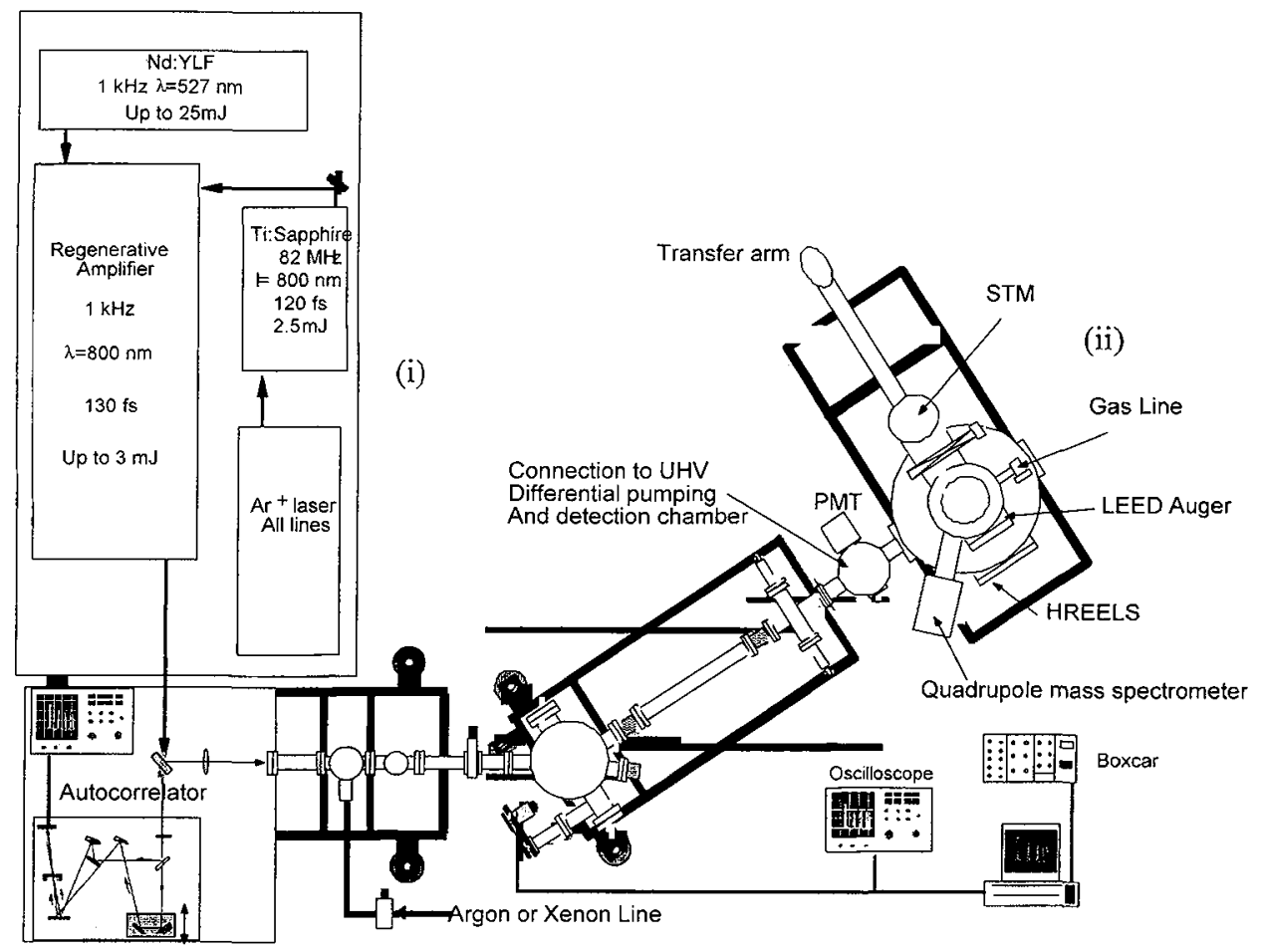

Figure 1: Schéma expérimental de la source XUV par HOHG et l'enceinte à vide pour l'étude de photodésorption.

Physics). L'ensemble permet d'obtenir $3 \mathrm{~mJ}$ à $800 \mathrm{~nm}$ pour une durée d'impulsion, après re-compression, de $130 \mathrm{fs}$. Ce faisceau infrarouge est focalisé par une lentille en quartz $(\mathrm{f}=50 \mathrm{~cm})$ afin d'obtenir un diamètre au plan focal d'environ $300 \mu \mathrm{m}$ dans la zone d'interaction avec le gaz rare. Cette zone est constituée d'un tube en acier de $3 \mathrm{~mm}$ de diamètre dans lequel circule du xénon ou de l'argon à différentes pressions. Il est muni d'un trou de $400 \mu \mathrm{m}$ de diamètre au travers duquel le faisceau fondamental se propage. Ce tube est fixé sur deux platines de translation permettant d'optimiser les positions de focalisation et l'accord de phase. L'ensemble est clos dans une enceinte à vide en pompage primaire $\left(10^{-4} \mathrm{mbar}\right)$. Cette configuration permet d'atteindre des intensités de $\mathrm{I}_{\mathrm{p}} \sim 1.610^{14} \mathrm{~W} / \mathrm{cm}^{2}$. Le faisceau fondamental et les faisceaux harmoniques se propagent collinéairement vers le réseau du monochromateur à travers un premier diaphragme permettant d'obtenir un vide différentiel dans la seconde enceinte sélectionnant les harmoniques $\left(\sim 10^{-8} \mathrm{mbar}\right)$. Le réseau utilisé est un réseau sphérique $(\mathrm{R}=3 \mathrm{~m})$ en silice de $1000 \mathrm{t} / \mathrm{mm}$ recouvert de platine $(400 \AA)$ travaillant à un angle de 71 degrés d'incidence. La rotation de ce réseau de \pm 7 degrés permet au monochromateur de sélectionner les harmoniques d'ordre 7 à l'infini sur la fente de sortie. La détection absolue des photons VUV est réalisée en observant la fluorescence d'un dépôt de sodium salicylate après la fente de sortie. L'efficacité quantique de ce dernier étant relativement constante [5] pour des excitations variant de $3.5 \mathrm{eV}$ à $35 \mathrm{eV}$.

(ii) Une troisième enceinte à vide, en pompage différentiel, est placée entre la sortie du monochromateur et l'enceinte ultra vide d'analyse ou règne un vide de $\sim 2.10^{-10}$ mbar. Cette dernière est équipée d'un spectromètre haute résolution à perte d'énergie électronique (HREELS), d'un spectromètre de masse quadripolaire, d'un écran pour la spectrométrie d'électrons lents (LEEDS) ainsi qu'un microscope à effet tunnel (STM) et son bras de transfert. L'échantillon est maintenu sur un support en cuivre ou molybdène et peut atteindre des températures de $26 \mathrm{~K}$. Une ligne de gaz fixée sur la partie supérieure de l'enceinte permet le dépôt de molécules sur le type de substrat choisi. 


\section{Etude expérimentale, résultats}

Les premiers tests permettant d'obtenir un spectre harmonique ont été réalisés dans le xénon afin de faciliter leur détection. La forte intensité infrarouge appliquée sur le jet de gaz induit une ionisation de ce dernier lors de processus multi-photoniques (MPI) et lors de processus par effet tunnel (TI)[6]. Le rapport d'ionisation de ces deux processus est souvent décrit par le paramètre de Keldysh [7] relatant la compétition entre MPI et TI lorsque la longueur d'onde du faisceau fondamental et sa durée d'impulsion varient. Dans nos conditions expérimentales l'ordre le plus élevé $N_{\max }$ que nous pouvons obtenir est décrit par la relation (1). Où hv $=1.5$ $\mathrm{eV}$ est l'énergie du photon infrarouge et $\mathrm{U}_{\mathrm{p}}$ est l'énergie pondéromotive de l'électron, également décrite comme l'énergie qu'il peut absorber (accélération) dans le champ laser après avoir été ionisé par effet tunnel.

$$
N_{\max }=\frac{I_{p}+3.17 U_{p}}{h v}
$$

$U_{p}$ est décrit par la relation (2) et le coefficient 3.17 est issu d'approximations théoriques [8].

$$
U_{p}=\frac{E^{2}}{4 \omega^{2}}
$$

L'ionisation par effet tunnel est améliorée pour de grandes longueurs d'ondes et pour de fortes intensités où $\gamma=\sqrt{I_{p} / 2 U_{p}}$ est inférieur à 1 . Il apparaît cependant, dans notre cas, que l'énergie de coupure expérimentale correspond à un coefficient 2 devant le terme $U_{p}$. Le xénon possédant un potentiel d'ionisation de $12.2 \mathrm{eV}$ nous pouvons, théoriquement, observer une coupure du plateau pour l'harmonique d'ordre $19(\sim 29.45 \mathrm{eV})$.

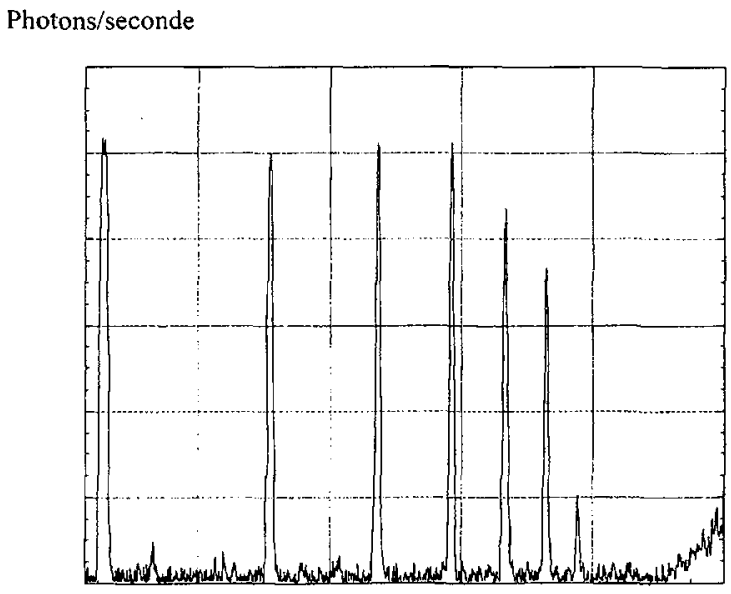

$\lambda(\mathrm{nm})$

Figure 2: Spectre Harmonique obtenus dans le xénon

Pour l'argon, la coupure est atteinte pour l'harmonique d'ordre $27(\sim 41.85 \mathrm{eV})$. La figure 2 représente un spectre harmonique lors de l'utilisation de xénon pour différentes pressions de gaz. Le spectre de la figure 2 a été obtenu pour une pression de 30 mbar. Lors de l'utilisation de l'argon, le spectre présente des émissions 
supplémentaires intermédiaires correspondant aux réflections des seconds et troisièmes ordre du réseau du monochromateur. La mesure absolue d'énergie à été réalisée en calibrant sous rayonnement UV un scintillateur en salicylate de sodium. Nous obtenons un flux de photons de $\sim 10^{7} \mathrm{ph} / \mathrm{tir}$ après le monochromateur.

\section{Systèmes physisorbés, applications}

Notre équipe à récemment découvert, lors d'études réalisées sous synchrotron, de nouveaux canaux de désorption [9] de $\mathrm{l}^{\prime} \mathrm{O}_{2}$ adsorbée sur du graphite (HOPG) en monochouche à $27 \mathrm{~K}$. Ces études relatent l'existence d'état de Rydberg $\left({ }^{2} \Pi_{u}\right)$ transitoire avant de produire l'état dissociatif $\left(O\left({ }^{3} \mathrm{P}\right)+\mathrm{O}^{-}\left({ }^{2} \mathrm{P}\right)\right)$ donnant lieu à la désorption brutale de $\mathrm{O}^{\circ}$ vers $15.3 \mathrm{eV}$. Ce système comparé à $\mathrm{CO} / \mathrm{HOPG}$ fait l'objet d'expériences actuelles où une compétition a lieu avec des phénomènes de désorption induite par le substrat (processus DIMET : Desorption Induced by Multiple Electrons Transition) particulièrement probables lors de l'utilisation d'impulsion ultra brève. Nous désirons également étudier des systèmes chimisorbés comme le $\mathrm{SF}_{6}$ ou le $\mathrm{CCl}_{4}$ sur des échantillons de silicium (100) (2x1). A température ambiante, le $\mathrm{SF}_{6}$ se dissocie en s'adsorbant sur le silicium et passive les doubles liaisons pendantes $\mathrm{S} i$. Une gravure spontanée peut alors avoir lieu lorsque la recombinaison de $\mathrm{SiF}_{2}+\mathrm{SiF}_{2}$ provoque la désorption de $\mathrm{SiF}_{4}+\mathrm{Si}$. L'étude de la photodésorption de ce type de système par irradiation UVX sous différentes conditions expérimentales en terme de température d'échantillon ou de longueur d'onde sera réalisé prochainement.

De plus, nous avons débuté la mesure du déclin de fluorescence de polymères organiques. Le poly-ethylène téréphtalate (PET) ou le poly-ethylène naphtalate $(\mathrm{PEN})$, étudié en électroluminescence pour les OLEDs (Optical Light Emiting Diodes), a été utilisé dans notre expérience comme scintillateur. Il fait l'objet actuellement d'une première application de démonstration en spectroscopie. Nous avons pu mesurer une dépendance non linéaire du déclin de fluorescence de ce scintillateur lors d'une excitation UVX variant de $112 \mathrm{~nm}$ à $30 \mathrm{~nm}$.

\section{Conclusion}

Nous avons décrit un système simple permettant d'obtenir une source cohérente UVX à impulsion brève dans la gamme d'énergie $11 \mathrm{eV}-42 \mathrm{eV}$ par génération d'harmoniques dans les gaz rares. Cette source, principalement dédiée à des expériences dans les domaines de la physique de la surface ouvre également de nouveaux potentiels d'études dans les domaines de la physique des matériaux et de la spectroscopie.

\section{Remerciements: EPSRC, EU}

\section{Références}

1. A. L'Huillier, T. Auguste, Ph. Balcou, B. Carré, P. Monnot, P. Salières, C. Altucci et al., K.S. Budil et al., J. Nonlinear. Opt. Phys. Mat. 4, 3 (1995).

2. C. Altucci, R. Bruzzese, C. de Lisio, M. Nisoli, S. de Silvestri, O. Svelto, A. Boscolo, P. Ceccherini, L. Poletto, G. Tondello, P. Villoresi, Phys. Rev. A. 6121601 (1999).

3. A. Sampera, Phys. Rev. A. 49, 3 (1994).

4. E. Constant, D. Gorzella, P. Berger, E. Mevel, Ch. Dorrer, C. Le Blanc, F. Salin, Agostini, Phys. Rev. Lett. 82, 8 (1999).

5. A. Knapp, A. M. Smith, Appl, Opt. 3, 5 (1964).

6. M. Levenstein, Ph. Balcou, M. Yu. Ivanov, A. L'Huillier, P. B. Corkum, Phys. Rev. A. 49, 3 (1994).

7. L.V. Keldysh, Sov. Phys. JETP 20, 5 (1965).

8. C.G. Vahlström, Phys. Scr. 49251 (1994).

9. L. Siller, S.L. Bennett, M.A. Mc. Donald, R.A. Bennett, R.E. Palmer and J.S. Foord, Phys. Rev. Lett., 76, 11 (1996). 